\title{
Das Corona-Dispositiv
}

\author{
Simon Duncker
}

\section{Einleitung}

Michel Foucaults Begriff Biomacht (vgl. Foucault 1977a: 159ff.) beschreibt eine spezifisch moderne Wissens- und Machtordnung, die das menschliche Leben zum Objekt hat. Dessen Steigerung, Vermehrung, Verbesserung und Nutzbarmachung ist ihr Ziel. Biomacht ist gemeinsam mit der kapitalistischen Produktionsweise entstanden, ermöglicht und stützt diese durch die Sicherung von Arbeitskraft. Kapitalakkumulation ermöglicht umgekehrt weitere Lebenssteigerung. Zielt der Begriff Biomacht zwar auf die wissenschaftliche und politische Ordnung der meisten gesellschaftlichen Felder, ist die Gesundheitsversorgung ein Paradebeispiel. Da ist es naheliegend, dass auch die Bekämpfung des SARS-CoV-2-Virus mit dem Biomacht-Konzept zu fassen versucht wird. Zwei zentrale Punkte scheinen mir dabei bisher zu kurz zu kommen: Erstens spielen neben biopolitischen Mechanismen der statistischen Bevölkerungsvermessung und -normalisierung immer auch Disziplinartechniken und Mechanismen der Souveränität ihre spezifischen Rollen für die Verwirklichung der Biomacht. Das zeigt sich insbesondere in Krisenzeiten wie diesen, wenn einstmals zurückgedrängte Mechanismen von der politischen Ordnungsmacht wieder ausgeweitet werden. Um das zu verdeutlichen, wird im ersten Abschnitt zunächst die Entwicklung des modernen Gesundheitsdispositivs skizziert (I.), um anschließend Maßnahmen der Pandemiebekämpfung exemplarisch nach Regierungsrationalitäten zu differenzieren (II.). Zweitens vernachlässigen bisherige Darstellungen die Biopolitische Grenzziehung, die ein konstitutives Moment jeder Biomacht darstellt und Gegenstand des dritten Kapitels sein wird (III.). Zäsuren zu Bevölkerungsgruppen, die dem Tod mit erhöhter Wahrscheinlichkeit legitim überlassen werden, sind in der Gesundheitsversorgung immer anwesend, erhalten jedoch in einer beispiellosen Krise wie dieser traurige Virulenz. Im letzten Abschnitt wird die Auffassung vertreten, dass eine Linke die tragische Sichtbarwerdung der Zäsuren dafür nutzen kann, einerseits nach der Universalisierung des biopolitischen Prinzips und andererseits nach der Aneignung der Produktivkräfte von Biomacht zu streben (IV.). 


\section{Das moderne Gesundheitsdispositiv und seine neoliberale Zurichtung}

Es war nie so, dass Biopolitik die Souveränitäts- und die Disziplinarmacht, in Differenz zu denen Foucault das Konzept in seinen Vorlesungen (vgl. Foucault 2005a; 2014) entwickelte, völlig verdrängt hätte. Um das zu erläutern, scheint es mir hilfreich zu sein, zwischen Biomacht und Biopolitik zu unterscheiden. Biomacht möchte ich durch ihren Gegenstand (Leben) und ihren Zweck (Leben steigern) bestimmen. Unter Biopolitik hingegen verstehe ich eine bestimmte Technik, die sich in den Dienst der Biomacht stellt: jene moderne Form politischer Regulierung, die die statistische Vermessung und Normalisierung von Bevölkerungsdynamiken verfolgt. Biopolitik beobachtet Gesetze, die dem Bevölkerungskörper eigen sind, und versucht diese einer statistischen Normalität anzunähern, indem sie für die Bevölkerung einen Regierungsraum aus Handlungsmöglichkeiten und -anreizen strukturiert, innerhalb dessen sich freie Subjekte bewegen. Diese politische Regulierung ist jedoch niemals die einzige Machttechnik, durch die sich Biomacht verwirklicht. Souveränitäts- und Disziplinarmacht wurden zwar zurückgedrängt, sind aber nie verschwunden, sondern haben im aufgeklärten Zeitalter der Biomacht ihre neuen Funktionen erhalten. Biopolitik, Disziplin und Souveränität haben sich in der Moderne zu einem Gewebe verknüpft, um der Biomacht zu dienen. Dieses Gewebe in Bezug auf die Kontrolle eines bestimmten Gegenstandes zu rekonstruieren, bedeutet, das Dispositiv dieses Gegenstandes zu beschreiben. Welche Maßnahmen sind als Techniken den einzelnen Machtformen zuzuordnen? Wie verknüpfen sich diese Techniken, um das Phänomen zu kontrollieren? Diese Differenzierungen und Verknüpfungen in Bezug auf den Umgang mit dem Corona-Virus vorzunehmen, bedeutet also, das Corona-Dispositiv zu beschreiben.

Die Gesundheitsversorgung war nie einzig Gegenstand biopolitischer Bestrebungen, sondern gründete sich von Anfang an auf die Disziplin der Spitäler. Ihre relative und spezifizierte Funktionsbestimmung innerhalb eines Regierungsraums aus medizinischen Praktiken und Institutionen war das Projekt der Staatsmedizin des 18. Jahrhunderts (vgl. Foucault 1988: 32ff.). Die flächendeckende Etablierung und Verknüpfung von Spitälern gehörte zu den ersten Maßnahmen biopolitischer Gesundheitspolitik der sich ausbildenden Nationalstaaten, die (medizinisch bestimmte) Gesundheitsnormen durchzusetzen versuchten (vgl. Foucault 1977b: 237ff.). Spitäler sind Disziplinarinstitutionen. Sie zielen auf den individuellen Körper als biologischen Organismus, den die moderne Medizin seit Bichat über seine Lebenserhaltungsprozesse definierte, durch die er einer todbringenden Umwelt eine Lebenszeit lang trotzt (vgl. Foucault 1988, 206ff.). Der 
Ärztliche Blick auf diesen Körper stellt das Individuum innerhalb der medizinischen Sprache her und ordnet es als Fall in einem institutionellen Raum an, der durch die Autorität des Arztes strukturiert ist (vgl. Foucault 1988: 12). Sein Wissen bestimmt die Regeln des Spitals, das die Körper überwacht, sie Anweisungen unterwirft und dadurch ein Verhalten wiederherstellt, das den als normal bestimmten Lebensprozessen des Körpers entspricht. Außerdem kommt dem Spital durch die Isolierung ansteckender Krankheiten eine Exklusionsfunktion zu. Im 19. Jahrhundert verbanden sich im Spital die Erfahrungen zweier Epidemien des 17. Jahrhunderts: Der Traum einer reinen Gemeinschaft, der sich in den radikalen Ausschlüssen der Leprosorien zeigte, und der während der Pest aufkommende Traum einer disziplinierten Gemeinschaft, die sich an Hygieneund Distanzregeln hält, um den Nächsten nicht zu gefährden (vgl. Foucault 1977b: 253ff.).

Das freie Subjekt, das sich selbstbestimmt durch den modernen biopolitischen Raum aus Gesundheitsdienstleistungen und -produkten außerhalb des Spitals bewegt, verfügt bereits über einen hinreichend disziplinierten Körper, der sich selbst versorgen kann und keine Gefahr für Andere darstellt. Die Biopolitik geht damit über die Koordinierung von Disziplinarinstitutionen - hier der Spitäler - hinaus. Gesundheitspolitische Regulierung hat mindestens drei Aufgaben: Erstens sammelt sie Daten über den Gesundheitszustand und die Bedarfe der Bevölkerung, um das Versorgungsnetz kalkulieren und bereitstellen zu können, das für die Herstellung der medizinischen Norm benötigt wird. Zweitens hat sie mit dem Vorsorgestaat ein solidarisches Versicherungssystem geschaffen, das gewährleistet, dass dieses Versorgungsnetz finanziert wird und idealerweise von jedem*r Bürger*in in Anspruch genommen werden kann. Dafür müssen Krankheiten und ihre Folgen statistisch als Risiken kalkuliert und diese Risiken nach einem Solidaritätsmodell vergemeinschaftet werden (vgl. Ewald 1993: 450ff.). Drittens wirkt Biopolitik präventiv (vgl. ebd.: 430f.): Sie sorgt präventiv für Existenzbedingungen, unter denen Krankheiten weniger wahrscheinlich auftreten und nähert so ihre Häufigkeiten einer statistischen Normalität auf der Ebene der Bevölkerung an, die durch ein Gesundheitssystem kurativ bewältigt werden kann, das die medizinische Norm auf individueller Ebene wiederherstellt. ${ }^{1}$

Die Macht medizinischer Disziplin und biopolitischer Gesundheitspolitik funktionieren produktiv: sie stellen Gesundheit her. Die Souveränitätsmacht hingegen funktioniert negativ, da sie Verhalten nicht fördert, son-

1 Über den Unterschied zwischen Normalität und Normativität (vgl. Link 2003). 
dern für den Bevölkerungskörper ungesundes Verhalten beschränkt. Das geschieht insbesondere durch gesetzliche Verbote. Im modernen Gesundheitsdispositiv, das sich im 19. Jahrhundert ausbildete, konnten Techniken der Souveränität weitestgehend zurückgedrängt werden. Sie beschränken sich heute auf einzelne Bereiche, zum Beispiel Kompetenzen von Gesundheitsämtern, Menschen mit hochansteckenden Krankheiten zu isolieren, Zwangsbehandlungen von als psychisch krank befundenen Menschen, denen Gerichte Selbstgefährdung bescheinigen, Rauchverbote oder eine kriminalisierende Drogenpolitik. Auch der disziplinarische Anteil ist geringer geworden, da Funktionen des Spitals durch ambulante und präventive Leistungen übernommen wurden. Der moderne Traum der Normalisierung und Kontrolle statistischer Risiken ist weder das Modell der Lepra (Ausschluss), noch der Pest (Disziplin), sondern nimmt sich den Umgang mit den Pocken zum Vorbild (Biopolitik), bei dem Bevölkerungsrisiken kalkuliert und präventiv (v.a. durch riesige Impfprogramme) verringert wurden (vgl. Sarasin 2020).

Dieses moderne Gesundheitssystem der Biomacht beruht auf einer starken Biopolitik. War diese durch ihre staatliche Solidaritäts- und Versicherungslogik lange marktwirtschaftlichen Mechanismen zumindest ein Stück weit entzogen, hat ihre neoliberale, in Deutschland insbesondere seit den Neunzigerjahren vorangetriebene Ökonomisierung einen Punkt überschritten, an dem Imperative der Kostenminimierung und -effektivität medizinische Versorgungsansprüche überformen (vgl. Bauer 2006). Gesundheitspolitische Datenakkumulation diente fortan der Kalkulation, wie weit staatliche Finanzierungen zurückgefahren werden können. Die DRGFallpauschale hat Einrichtungen dazu gezwungen, nach marktwirtschaftlichen Kriterien zu handeln, die medizinischen Normen widersprechen können. Das solidarische Versicherungssystem wurde untergraben, indem Leistungen staatlicher Krankenkassen nachgelassen haben und private ( $\mathrm{Zu}$ satz-)Versicherungen dadurch für (neo-)liberale Subjekte, die Mittel nach individuellen Zwecken abwägen, rational wurden. Die Prävention setzte nicht auf die Bereitstellung von gesundheitsfördernden Existenzbedingungen durch staatliche Maßnahmen (wozu großzügige Sozial- und Arbeitsrechte zählen), sondern auf die Eigenverantwortung der Bürger*innen, die ständig Gefahr laufen, für einen Teil ihrer Behandlung selbst aufkommen zu müssen. Das erzeugt ein individuelles Interesse oder sogar Druck, seinen Beitrag zur nationalen Gesundheit zu leisten. Einen Appell zur Volksgesundheit braucht Biopolitik nicht mehr. Der Effekt der neoliberalen Maßnahmen war eine Herabsetzung der Kapazitätsgrenze des Gesundheitssystems, die auf unsichere Strategien der Normalisierung baut und 
ständig überschritten zu werden droht, sodass medizinische Normen in bestimmten Sektoren nicht mehr gewährleistet werden können.

\section{Das Gesundheitsdispositiv in der Corona-Krise}

Der Biopolitikbegriff kann nun nicht pauschal auf die Corona-Krise angewendet werden. Philipp Sarasin ist darin zuzustimmen, dass differenziert werden muss, auf welche historischen Modelle der Epidemiebekämpfung (Lepra, Pest, Pocken) zurückgegriffen wird. An dem Umgang mit Corona war weniger bemerkenswert, wie Regierungen zu den üblichen biopolitischen Mechanismen greifen: Erhebung von Infektionen, kritischen Fällen, Sterberaten, Kalkulation von künftigen Entwicklungen, Abschätzen des Bedarfs an Intensivbetten, Personal, Gesundheitsprodukten etc. sowie die (z.T. unzureichende) Bereitstellung benötigter Ressourcen. Das Bemerkenswerte war vielmehr, dass offenbart wurde, wie ein Staat reagiert, wenn seine neoliberal zugerichtete Biopolitik ihre Funktion im Gesundheitsdispositiv nicht mehr bewältigen kann. Schon Vermessung und Kalkulation gestalteten sich schwierig, unter anderem deshalb, weil das Ausmaß der Krise (zumindest für Industriestaaten in Friedenszeiten) völlig neu, und Testkapazitäten stark limitiert waren. Auf internationaler Ebene hat die Sammlung vergleichbarer Daten und ihr Austausch erst recht nicht funktioniert, da die Gesundheitssysteme und ihre datensammelnden Behörden wie das Robert-Koch-Institut national organisiert sind und Testpraktiken wie Rahmenbedingungen zu unterschiedlich waren.

Die Denormalisierung der Bedarfskurven (im doppelten Sinne: sie sind schlicht unbekannt, aber mutmaßlich über den Kapazitäten), die Alex Demirović beobachtet (vgl. Demirović 2020), führte meines Erachtens zu einer Wiederausweitung von durch Biopolitik vormals verdrängten Machtmechanismen, um die Sicherheit der Bevölkerung gewährleisten und ihre Arbeitskraft erhalten zu können. Es ist also nicht so, wie Paul B. Preciado annimmt, dass die hegemonialen politischen Rationalitäten der Biomacht den Umgang mit Pandemien determinieren. ${ }^{2}$ Vielmehr werden ihre Ausprägungen und Verhältnisse zueinander restrukturiert. ${ }^{3}$

2 „Tell me how your community constructs its political sovereignty and I will tell you what forms your plagues will take and how you will confront them" (Preciado 2020).

3 Vielleicht laufen biopolitische Strukturen des Vorsorgestaats wie die des Gesundheitssystems im neoliberalen Zeitalter ständig Gefahr, wieder durch einen stärker autoritär agierenden Staat kompensiert werden zu müssen. Falls Corona dem Neo- 
Dies geschah zum einen durch die Ausweitung souveräner Befugnisse. Bei den weltweiten Einschränkungen der Bewegungs-, Versammlungs-, Gewerbe-, Berufs- und Religionsfreiheit handelt es sich offensichtlich nicht um Biopolitik im hier dargestellten Sinne, auch nicht um eine Mischung aus Disziplin und Biopolitik, wie Precario annimmt, sondern um die harte Hand eines Souveräns, der mit Verboten und Repression unerwünschtes Verhalten, das dem Zweck der Biomacht (hier Erhaltung der Gesundheit der Bevölkerung) entgegensteht, zu verhindern sucht. Reaktionen der Souveränität auf Covid-19 sind nicht autoritären Herrschern illiberaler Demokratien (Putin, Orban) und solchen, die es gerne wären (Trump), vorbehalten, sondern zeigen sich in fast allen betroffenen Ländern. Das Infektionsschutzgesetz machte eine in der BRD bisher einmalige Einschränkung von Grundrechten möglich, bei der sonst übliche verfassungsrechtliche Abwägungen von Alternativen und Verhältnismäßigkeiten zu Beginn wenig stattfanden (vgl. Lepsius 2020). In neoliberaler Rhetorik der Alternativlosigkeit versteckte der Souverän den politischen Charakter seiner epidemiologisch rationalen Repression. Die Schließung und Kontrolle nationaler Grenzen ist oberstes Recht des Souveräns und keine direkt biopolitische Maßnahme. Biopolitik versucht freie Entscheidungen zu kalkulieren und (un-)wahrscheinlich zu machen, anstatt Freiheiten zu beschränken. Um die Kapazitäten der Biopolitik nicht zu überlasten und ihre Normalisierungskurve abzuflachen, ergreift der Staat also ihr fremde Maßnahmen, die die Gesundheitspolitik anderer Jahrhunderte kennzeichnete.

Welche Rolle nimmt zum anderen die Disziplin im Corona-Dispositiv ein? Sie war die erste Machtform, die die Souveränitätsmacht im 17. und 18. Jahrhundert zurückdrängte (vgl. Foucault 2014: 91ff.). Würde nicht auch sie die erste sein, die sich in Krisenzeiten wieder aufdrängt? Doch auf welche Institutionen soll sich gesundheitspolitische Disziplin schon stützen? Fabriken und Schulen waren geschlossen. Der Anteil, den Krankenhäuser an der Gesundheitsversorgung haben, ist längst zugunsten anderer, niedrigschwelligerer Gesundheitsdienstleistungen zurückgegangen. Zu Zeiten der Denormalisierung durch Corona drohten die Krankenhäuser deshalb überlastet zu werden und ihrer Doppelfunktion (Ausschluss und Behandlung) nicht ausreichend nachkommen zu können. Das Corona-Dispositiv ist daher auf eine disziplinierte Bevölkerung angewiesen, deren Individuen ihre Bewegungen von selbst kontrollieren (über-

liberalismus nicht sein Grab schaufelt, wie nun viele Linke durch die eiligen keynesianischen Interventionen hoffen, dürfen wir vermuten, wozu Staaten bei Krisen in anderen Bereichen fähig sein werden. 
flüssige Wege und Kontakte vermeiden), Hygienebestimmungen befolgen und sich im Falle einer möglichen Erkrankung von selbst zu Hause isolieren. Eine ,disziplinierende ' Wirkung der Spitäler nach außen besteht allenfalls noch in der Drohung, den Zumutungen eines Krankenhausaufenthalts in Krisenzeiten ausgeliefert zu sein. Doch Disziplinierung im Sinne der Anwendung der für sie typischen Techniken (vgl. Foucault 1977b: 173ff.) ist außerhalb der dafür vorgesehenen Institutionen nur schwerlich möglich. Denn sie erfordert Möglichkeiten ständiger Überwachung individueller Körper und ihrer potenziellen Sanktionierung. Vom isolierten Homeoffice als digital vernetztem neuen Hort der Disziplin zu reden, wie es Preciado tut (vgl. Preciado 2020), verwischt deshalb vollkommen die Spezifität des Disziplinbegriffs. Auch bei der Verfolgung von Körperbewegungen durch Smartphonedaten sollte Preciado nicht vorschnell auf eine Disziplinartechnologie schließen. Das wäre nur dann sinnvoll, sofern diese Daten individualisiert, zentralisiert und Anlass für individuelle Sanktionierungen werden können. Das ist vorerst nur in China der Fall, wo individuelle QR-Codes über Bewegungsfreiheiten entscheiden, Bewegungsprofile von der Polizei eingesehen werden können und es denkbar ist, künftig das Pandemieverhalten mit dem Punktesystem zu sanktionieren, durch das sich ,gute Bürger*innen' gegenseitig bewerten können. In liberalen Demokratien ist eine Restauration der Disziplin hingegen insgesamt schwierig.

Möchten Regierungen souveräne Verbote und deren wirtschaftlichen Schaden vermeiden, sind sie deshalb auf eine starke Biopolitik angewiesen. Länder wie Singapur und Südkorea konnten einen frühen Shutdown vermeiden, weil sie sich schnell präventiver biopolitischer Strategien bedienten (ausgiebige Testpraxis, Desinfektionsmittel an öffentlichen Orten, Tracing-App, gezielte Isolierung). Diese Strategien setzen aber hinreichend disziplinierte Subjekte voraus, die selbstbestimmt mitspielen. Eine Alternative einer ebenso individualisierenden Macht, die die vermassende Bevölkerungsregulierung der Biopolitik ergänzt, bietet die Pastoralmacht (vgl. Foucault 1977a: 139ff.) Das Pastorat funktioniert wie die Disziplin über Autoritäten, braucht aber keine klar umrissene Institution, in der die Autorität wirken kann, und weder Überwachung noch Bestrafung. Lieber hört es sich die Verfehlungen geständiger Individuen an, um ihnen den Weg zum Glück zu zeigen. Es konditioniert keine Körper, sondern appelliert an das Gewissen der Einzelnen, die es sanft zu führen weiß. Paradigmatisch lässt sich diese Machtform an der Ansprache der deutschen Bundeskanzlerin vom 18.3.20 verdeutlichen, deren Appell an staatsbürgerliche Disziplin und Solidarität immer wieder wiederholt wurde und im Gegensatz zur 
Kriegsrhetorik des Souveräns ${ }^{4}$ steht (Macron: Wir sind im Krieg!). Geständnisse brauchte es nicht. Die Einzelnen werden erkannt haben, ob sie gemeint waren - am schlechten Gewissen, das Besserung verlangt. Man solle doch seine Liebsten nicht gefährden und Distanz wahren. Überhaupt solle man ein*e gute*r Bürger*in sein und durch Einhaltung von Vorsichtsmaßnahmen dazu beitragen, die Kurve flach zu halten: Du, ja Du, bist Teil einer großen nationalen Aufgabe, der größten seit dem Zweiten Weltkrieg. Hier wird ein Subjekt angerufen, das seiner individuellen Verantwortung für seine Nächsten, besonders aber für seinen Fernsten (der als Teil derselben Nation wieder nah erscheint) nachkommt. Gesundheitspolitische Kontrolle und individuelle Verantwortung werden durch den Bezug auf die Kurve direkt miteinander verknüpft.

Diese Abstimmung zwischen Pastorat und Biopolitik, Selbst- und Fremdsteuerung ist das, was für Foucault den Fürsorgestaat kennzeichnet (vgl. Foucault 2005b: 199). Die Tracing-Apps, die in Ländern wie Singapur und Südkorea aufgrund geringerer datenschutzrechtlicher Hürden und fortgeschrittener Digitalisierung früh verfügbar waren, sind biopolitische Instrumente, die gewissenhafte Bürger"innen mit Informationen versorgen, damit sie ihre freien Bewegungen im biopolitischen Raum selbstbestimmt einschränken und lenken. Räume mit erwartungsgemäß erhöhter Ansteckungswahrscheinlichkeit können gemieden werden. Auch die deutsche Corona-Warn-App ist der Versuch, die Infektionskurve über digital gestützte Selbststeuerung biopolitisch zu normalisieren, indem mehr Fälle erfasst und Kontakte mit infizierten Personen zu einer selbstauferlegten Quarantäne veranlassen. Biopolitische Normalisierung durch Fremdsteuerung, das heißt die politische Vermessung, Planung und Strukturierung des gesundheitspolitischen Regierungsraums, ist da langwieriger. Die Heinsberg-Studie (vgl. Streeck 2020) gibt eine ungefähre Vorstellung von Dunkelziffern, Todesraten und Ansteckungsrisiken in Haushalten, ist aber aufgrund mangelnder Repräsentativität weit davon entfernt, seriöse Aussagen für nationale Politik treffen zu können. Die erste Studie, die repräsentative Schlüsse auf den deutschen Bevölkerungsanteil zulässt, der durch Antikörper (vermutlich) geschützt ist, ist erst für September 2020 geplant.

$4 \mathrm{Zu}$ der Verwendung von Kriegsmetaphern in der Corona-Krise, siehe den Beitrag von Malte Pasler in diesem Band. 


\section{Grenzen der Solidarität: Biopolitische Zäsuren}

Hätte das Wörtchen Solidarität doch nicht dem nationalen Zusammenrücken gedient, sondern uns mit dem Nächsten verbunden, für den wir gemeinsam Sorge tragen, und einem Ferneren, etwa in der italienischen Lombardei und im Flüchtlingslager Moria auf Lesbos. Denn hier, in der Entscheidung der Ärztinnen und Ärzte, welche*r Patient*in an die knappen Beatmungsgeräte angeschlossen wird, und dort, an den Rändern Europas, beobachten wir die biopolitischen Zäsuren, die Foucault zufolge jeder Biopolitik innewohnen. Mit "Staatsrassismus" (Foucault 2014: 111) bezeichnet er eine neue Funktion, die rassistische Vorurteile ab dem 18. Jahrhundert für die Etablierung der Biomacht erhalten. Diese darf durch ihren Zweck, Leben zu erhalten und zu mehren nämlich partout nicht direkt töten, wie es noch die Souveränitätsmacht konnte. Ihr Zugriff auf Leben und Tod erfolgt indirekt, durch den Ausschluss bestimmter Gruppen von biopolitischen Maßnahmen. Diese „Zäsur zwischen dem, was leben, und dem, was sterben muß“ (ebd.: 104) stellt eine positive Beziehung zwischen dem In-den-Tod-Stoßen der Ausgeschlossenen und den Lebenschancen einer Bevölkerung als Ganzer her. Das Konzept taugt für das historischspezifische Verständnis von Rassismus nur sehr bedingt, trifft aber einen Punkt. Es zeigt, wie Diskriminierungsmechanismen eine Funktion für Biomacht spielen (vgl. Sarasin 2003). Außerdem gehen bei Foucault zwei Zäsuren durcheinander: eine absolute Grenze, die eine Personengruppe gänzlich aus dem biopolitischen Kollektiv ausschließt, indem ihre Souveränität nicht anerkannt wird; und eine inhärente Grenze durch den Bevölkerungskörper des „biologischen Kontinuums, an das sich die Biomacht wendet“ (Foucault 2014: 104, Herv. i. O.). Diese Grenze setzt Bevölkerungsgruppen mit bestimmten Merkmalen dem Tod mit höherer Wahrscheinlichkeit aus. Das sind zwei sehr verschiedene Dinge, deren Differenz auch in der Corona-Krise zu beobachten ist.

Die absolute Grenze offenbart und symbolisiert sich in Moria. Die Figur des Flüchtlings verkörpert den Widerspruch zwischen Menschenrechten, die jedem Menschen als Lebewesen zukommen, und der nationalstaatlichen Verfasstheit von Biopolitik, die sich zwar auf das Lebewesen richtet, aber eine durch Pass oder zumindest Aufenthaltserlaubnis verbriefte (Teil-)Souveränität als Rechtssubjekt (Bürger*in) voraussetzt (vgl. Agamben 2014, 199ff.). Das Asylrecht bot zumindest eine Chance für Menschen, deren Anerkennung als Rechtssubjekt oder deren Erhaltung als Lebewesen in unmittelbarer Gefahr ist, Teil eines biopolitischen Kollektivs in Europa zu werden. War das Menschenrecht auf Asyl schon spätestens mit dem EUTürkei-Deal von 2016 bis zur Unkenntlichkeit untergraben, wurde es in 
Corona-Zeiten offiziell ausgesetzt. Selbst bezüglich der Evakuierung einer Handvoll unbegleiteter Kinder wurde gefeilscht. Denn man wollte sie ja gerade nicht haben. Die neo-souveränen Nationalstaaten ziehen die absolute Zäsur nun radikal, undurchlässig und explizit. Indem in den Legitimationen allen Ernstes ein positiver Zusammenhang zwischen dem Überleben der eigenen Bevölkerungen und dem Ausschluss von ein paar tausend Menschen konstruiert wird, die notfalls dem Verrecken preisgegeben werden, zeigt die Biomacht, die nach innen freundlicher dreinschaut, nach außen hin unverhohlener als sonst ihre hässliche Fratze. Während deutsche Urlauber*innen in die heimischen Gesundheitssysteme geflogen wurden, verschlimmerten sich die Lebens- und Sterbebedingungen in den Camps zunehmend.

Die inhärenten Grenzen sind weniger materiell und offensichtlich, anwesend bloß in der abstrakten Form lebensfördernder wie todbringender Wahrscheinlichkeiten. Jede Diskriminierung in der Gesundheitsversorgung setzt solche Grenzen, die immer dort relevant werden, wo auf knappe Ressourcen potenziell mehr Bedarf kommt. In der Corona-Krise zeigt sie sich am unmittelbarsten zwischen zwei an Covid-19 erkrankten Bürger*innen mit formell gleicher Berechtigung zur Gesundheitsversorgung, die um ein Beatmungsgerät konkurrieren. Die Ärzt"innen, die in der Lombardei schon früh unmögliche Entscheidungen über Leben und Tod treffen mussten, gerieten aufgrund praktischer Anforderungen in Konflikt mit der universellen Norm ihres Berufstandes (Hyppokratischer Eid). Die höchst unpersönliche Biomacht gerät in Konflikt mit sich selbst, da sie jede*n Bürger*in gleich als Lebewesen betrachtet, ihren Anspruch aber effektiv nicht einlöst. Menschliches Leben darf nicht gegeneinander aufgewogen werden. Andernorts wurden Normen für eine offensichtlich gewordene biopolitische Zäsur etabliert, die sich normalerweise lieber still und heimlich vollzieht. Im Elsass durften Menschen über 80 Jahren zeitweise nur noch auf Sterbebegleitung, nicht aber auf Beatmung hoffen. In manch anderen Kliniken Frankreichs galt 75 Jahre als Marke (vgl. Soldt/Wiegel 2020).

In Deutschland gab man sich in weiser Voraussicht Mühe, die unmögliche Entscheidung biopolitischer Grenzziehung zu rationalisieren. Die Deutsche Interdisziplinäre Vereinigung für Intensiv- und Notfallmedizin (DIVI) benannte zuerst im März 2020 Kriterien für Handlungsempfehlungen, die den Gleichheitsgrundsatz erhalten sollen und sich an der Überlebenswahrscheinlichkeit orientieren. Der Vorsatz, dass Alter, Behinderung, Herkunft und soziale Stellung keine Rolle spielen dürfen, ist löblich, kann aber schlecht verhindern, dass diese Merkmale effektiv wirken, weil sie Genesungswahrscheinlichkeiten und dadurch indirekt die Kriterien beein- 
flussen, die Rückschlüsse auf Wahrscheinlichkeiten geben sollen. Kalendarisches Alter und soziale Stellung dürfe nur nicht allein ausschlaggebend sein (vgl. DIVI 2020a: 4). Behindertenvertretungen (vgl. AbilityWatch 2020; NW3/ISL 2020) haben zügig kritisiert, dass Kriterien wie „allgemeiner Gesundheitsstatus“, durch eine Skala abgeschätzte „erhöhte Gebrechlichkeit“, bestimmte Nebenerkrankungen und die "Langzeitprognose“ pauschal Menschen mit bestimmten Behinderungen und alte Menschen benachteiligt. Die DIVI veröffentlichte im April eine zweite Version (vgl. DIVI 2020b), die die Kritik teilweise aufnahm, kann aber einen Grundkonflikt nicht lösen: Jedes Kriterium, das Rückschlüsse auf eine Wahrscheinlichkeit gibt, auch rein medizinische wie die Nebenerkrankungen oder der SOFA-Score, der die Wahrscheinlichkeit von Organversagen abschätzt, abstrahiert von individuellen Fällen. Der humanistische Gleichheitsgrundsatz kann sich nur aufgrund einer stochastischen Abstraktion behaupten, nämlich über die Feststellung statistischer Ungleichheit durch vergleichbare Merkmale. ${ }^{5}$ Gleichheitsgrundsatz hin oder her: auch ein Wahrscheinlichkeitskalkül zieht eine biopolitische Zäsur, indem ein positiver Zusammenhang zwischen dem Ableben einer Gruppe von Menschen und der Aufrechterhaltung der Gesundheit der Gesamtbevölkerung hergestellt wird, deren Mortalitätsrate durch rationale Ressourcenverteilung gedrückt werden soll.

Lässt sich die biopolitische Zäsur ökonomisch bestimmen? Im Vergleich zur Schweiz oder den USA wurden Debatten, die das Recht auf Leben mit ökonomischen Folgen in ein Verhältnis setzt - von Ausnahmen abgesehen - in Deutschland erst Ende April im Zuge der Diskussion um eine ExitStrategie breit geführt. Der konservative Rebell der Grünen, Boris Palmer, und Rechenmeister Wolfgang Schäuble provozierten großen Protest. Was bei Schäuble zumindest noch den Charakter einer Abwägung von Grundrechten hatte, bewegte sich bei Palmer nicht mehr auf dem Boden des Grundgesetzes, da er im Sat.1-Frühstücksfernsehen suggerierte, ein kürzeres Leben wäre weniger erhaltenswert. Doch auch der Deutsche Ethikrat

5 Der 81-jährigen Rentnerin würde die Beatmung nicht verweigert, weil sie als Individuum eine festgelegte Altersnorm überschreitet, sondern weil sie als Merkmalsträgerin eines im Vergleich geringeren Blutgerinnungswerts oder eine erhöhte Gebrechlichkeit der Stufe 7 aufweist, weil sie im Alltag auf Pflege angewiesen ist. Vor dem statistischen Orakel sind wir zwar alle so gleich wie vor Gott und dem Humanismus. Nach seinem Urteilsspruch sind wir es nicht. Über allgemeine Handlungskriterien für Ärztinnen und Ärzte bewahrheitet sich ein wahrscheinliches Schicksal, das in den Händen derer liegt, die humanistische Ideale verkörpern und nicht zwischen Menschenleben abwägen dürfen. 
sieht die Notwendigkeit einer Abwägung zwischen gesundheitspolitischen Zielen und „ökonomischen, kulturellen, politischen und psychosozialen Schäden“ (Deutscher Ethikrat 2020). Das Aufwiegen quantifizierter Menschenleben untereinander mit ökonomischen Interessen ist Teil jeder Gesundheitspolitik mit begrenzten Ressourcen. Jede Biopolitik strukturiert Lebens- und Sterbebedingungen von Merkmalsgruppen. Auch jede politische Entscheidung darüber, welche gesellschaftlichen Aktivitäten eingeschränkt werden, verschob von Anfang an die biopolitische Grenze. So betrifft auch die Ausgestaltung jeder Exitstrategie und die Bestimmung ihres richtigen Zeitpunkts die schlecht kalkulierbare Erwägung, der Tod wie vieler Menschen aus Risikogruppen mit einer wie großen Rezession aufgewogen werden kann, von der wiederum zukünftige Lebens- und Sterbebedingungen anderer Bevölkerungsgruppen betroffen sein werden. Jede Pandemiestrategie rechnet mit ihren Opfern. Wie groß die Opferbereitschaft ist und wie die Risiken verteilt werden, wird nicht zuletzt in der politischen Öffentlichkeit verhandelt. Großzügig gezogene Grenzen eines Trumps oder Bolsonaros sind keine Anzeichen für eine Abkehr von Biomacht, wie Sarasin annimmt. Unter kapitalistischen Bedingungen bleiben Biopolitik und Kapitalakkumulation direkt aneinandergebunden. Kapitalismus tötet, weil er - ob im Inland oder im Ausland - einen Mangel erzeugt, der verwaltet werden will. Wie, wie viele und wen er tötet, bestimmen biopolitische Mechanismen. An einer dynamischen Grenze kippt moderne Biopolitik deshalb immer in Thanatopolitik (vgl. Agamben 2014: 194).

\section{Globale Biopolitik und Biopolitik von Unten}

Biopolitik kann unter kapitalistischen Bedingungen den eigenen universellen Werten, die sie allen Lebewesen als Gleiche predigt, durch die beschriebenen inhärenten und absoluten biopolitischen Grenzen strukturell gar nicht gerecht werden. Mit Berufung auf dieselben Werte muss man ihr trotzdem jedes Lebewesen, das sie auf dem Gewissen hat, um die Ohren hauen, damit die Biomacht in Widerspruch mit sich selbst gerät. So spricht man dieselbe humanistische Sprache, die die biopolitischen Grenzen zurückdrängen kann. In Krisenzeiten wird das biopolitische Unvermögen überdeutlich, weil die absoluten Zäsuren schärfer und die inhärenten Zäsuren unsicherer werden. Es laut zu thematisieren, kann einer Doppelstrategie dienen: erstens der tendenziellen Universalisierung der Biopolitik von Oben und zweitens der Ermächtigung einer Biopolitik von Unten (vgl. Sotiris 2020). 
Das Streben nach der unmöglichen Universalisierung des biopolitischen Prinzips bedeutet erstens, die absolute Grenze so weit wie möglich nach außen hin zu verschieben und so durchlässig wie möglich werden zu lassen. Es ist die Forderung, möglichst viele Menschen an den Vorzügen biopolitischer Existenzsicherung teilhaben zu lassen. Dafür ist eine europäische Gesundheits- und Sozialpolitik unabdingbar, die Nationalismen überwindet und perspektivisch nach einer globalen Biopolitik strebt, in der jeder Mensch als Lebewesen zu seinem Recht kommt. Diese Universalisierung bedeutet zweitens, die Diskriminierungsmechanismen in der Gesundheitsversorgung, die die inhärenten Grenzen konstituieren, zu skandalisieren. Das ist in einer Krisensituation, in der ungewöhnlich viele Menschen von der Unsicherheit betroffen sind, einer höheren Sterbewahrscheinlichkeit ausgesetzt zu werden, vielversprechender als sonst. Jede"r kennt Menschen aus Risikogruppen. Wenn offensichtlich wird, dass Marktimperative nicht dazu in der Lage sind, für die Gesundheit aller zu sorgen, könnte das dazu beitragen, neue und alte Rahmenbedingungen für eine ausreichende und sichere Finanzierung des Gesundheitssystems zu erzwingen.

Biopolitik für alle einzufordern, bedeutet anzuerkennen, dass sie zwar problematisiert werden muss, aber die bisher erstrebenswerteste Machtform darstellt. Ihre Ausweitung durch den Vorsorgestaat, die mit einem Zurückdrängen anderer Machtformen verbunden war, ist eine Errungenschaft. Ihre Rationalität einer liberalen Regierung (Gouvernementalität) ermöglichte im 19. und 20. Jahrhundert den bislang größten Zuwachs individueller Freiheit und kollektiver Sicherheit. Die Ausweitung anderer Machtformen in der Corona-Krise führte uns das wieder vor Augen. Die Betrachtung der Gesundheitsversorgung mit Foucaults nivellierendem Machtbegriff sollte uns auf die Anerkennung dieser Vorzüge verpflichten. Giorgio Agambens Kritik an den italienischen Regierungsmaßnahmen (vgl. Agamben 2020) ist seiner Philosophie des Homo Sacer geschuldet, die dies nicht leisten kann und dadurch einen antimodernen Einschlag erhält. Die Universalisierung von Biopolitik ist für ihn der Skandal eines totalitären Staates, dessen Zugriff auf das menschliche Leben totalitär geworden ist. Ist die Problematisierung einer Tendenz massenweiser Verwaltung von menschlichem Leben zwar richtig, verkennt jedoch seine Einseitigkeit die moderne Dialektik, die durch Kontrolle und Sicherheit auch Freiheit ermöglichte. Seine Behauptung, dass die biopolitische Grenze in der Moderne durch uns alle geht, wir alle zu nacktem, tötbarem Leben werden, verwischt im selben Zug alle tatsächlichen inhärenten und absoluten Grenzen von Bevölkerung und erklärt mit großem Pathos einen permanenten Ausnabmezustand für alle. Nur aufgrund der Projektion seiner Theorie auf 
einen tatsächlich erklärten Ausnahmezustand ${ }^{6}$ kann er ernsthaft den Menschen raten, mal weniger Angst vor dem Sterben zu haben und gegen die staatlichen Einschränkungen des guten Lebens zu opponieren. Doch wir sind nicht alle gleich vor Corona. Die Grenzen zu denen, die im Zweifel geopfert werden, nimmt er zwar wahr, kann sie aber theoretisch nicht inkorporieren.

Die Universalisierung von Biopolitik ist nicht genug. Sie bleibt eine Biopolitik von Oben, die nicht über das sozialdemokratische Ideal eines halbwegs funktionierenden Für- bzw. Vorsorgestaats unter kapitalistischen Produktionsbedingungen hinausweist. Deshalb ist die Universalisierung gleichzeitig mit einer Biopolitik von Unten zu verknüpfen, die sich die Ressourcen aneignet, die Staaten wieder bereitzustellen gezwungen werden. Das bedeutet erstens, darauf zu drängen, dass die Verwaltung der Produktivkräfte im Gesundheitssektor durch demokratische und dezentrale Strukturen der Arbeitnehmer*innen selbst erfolgt. Dafür bedarf es u.a. basisorientierte Gewerkschaftsarbeit. Auch die Daten des biopolitischen Raums müssen der Kontrolle derjenigen unterstehen, deren Handeln objektifiziert wird. Sie sind die Produzent"innen ihrer Daten. Biopolitik von Unten bedeutet zweitens, Strukturen zu fördern und zu verstetigen, die weder privatwirtschaftlicher noch staatlicher Art sind, sondern Sorgearbeit und Prävention auf solidarischer Basis organisieren. Dazu zählen u.a. Nachbarschafts- und Stadtteilinitiativen sowie soziale Bewegungen.

Biopolitik von Unten ist also völlig anderen Akteuren mit z.T. widersprüchlichen Strategien und Zielen zuzuordnen und unterscheidet sich von der Biopolitik von Oben durch ein negatives Verhältnis zum Staat, auf den sie aber angewiesen bleibt. Ausweitung und Enteignung staatlicher Kompetenzen zwecks ihrer basisdemokratischen Verwaltung ist die gleichzeitig zu forcierende widersprüchliche Doppelbewegung emanzipatorischer Biopolitik. In einer krisenevozierten Organisation eines biopolitischen Sektors von unten, die sich in der Befriedigung grundlegendster Bedürfnisse bewährt, könnte der Keim einer nicht-staatszentrierten sozialistischen Biopolitik liegen, der über das Bestehende im Ganzen hinausweist. Die Alternative eines autoritär agierenden Staates, der Biopolitik von Oben forciert und exklusiv einschränkt, ist angesichts wirtschaftlicher und politischer Krisenfolgen in vielen Staaten Europas leider nicht unwahrscheinlicher. $^{7}$

6 Demokrat Ramadani deutet in seinem Beitrag für diesen Band die Corona-Krise als Ausnahmezustand für und durch die Demokratie.

7 Dieser Text ist mit Unterstützung der Gerda Henkel Stiftung entstanden. 


\section{Literaturverzeichnis}

AbilityWatch. 2020. Fachgesellschaften veröffentlichen ethisch und verfassungsrechtlich fragwürdige COVID19-Empfehlungen. https://is.gd/FF9LN6. 30.3.2020.

Agamben, Giorgio. 2014. Homo Sacer: Die souveräne Macht und das nackte Leben. In: Andreas Folkers/Thomas Lemke (Hrsg.), Biopolitik. Ein Reader. Berlin: Suhrkamp, 191-227.

Agamben, Giorgio. 2020. Nach Corona: Wir sind nunmehr das nackte Leben. NZZ. https://is.gd/YnKguj.

Bauer, Ullrich. 2006. Die sozialen Kosten der Ökonomisierung von Gesundheit. Aus Politik und Zeitgeschichte 8-9: 17-24. https:/www.bpb.de/system/files/pdf/9JE AEH.pdf.

Deutscher Ethikrat. 2020. Solidarität und Verantwortung in der Corona-Krise. Ad-HocEmpfehlungen. https://is.gd/W3RH3F. 27.03.2020.

DIVI. 2020a. Entscheidungen über die Zuteilung von Ressourcen in der Notfall- und der Intensivmedizin im Kontext der COVID-19 Pandemie. https://is.gd/3 B maI6. 17.08.2020.

DIVI. 2020b. Entscheidungen über die Zuteilung intensivmedizinischer Ressourcen im Kontext der COVID-19-Pandemie. Version 2. https://is.gd/V4aFnJ. 17.08.2020.

Demirović, Alex. 2020. In der Krise die Weichen stellen. Die Corona-Pandemie und die Perspektiven der Transformation. LUXEMBURG ONLINE. https://is.gd/ v6Mygg.

Ewald, Francois. 1993. Der Vorsorgestaat. Frankfurt/M.: Suhrkamp.

Foucault, Michel. 1977a. Sexualität und Wabrheit. Erster Band: Der Wille zum Wissen. Frankfurt/M.: Suhrkamp.

Foucault, Michel. 1977b. Überwachen und Strafen. Die Geburt des Gefängnisses. Frankfurt/M.: Suhrkamp.

Foucault, Michel. 1988. Die Geburt der Klinik. Eine Archäologie des ärztlichen Blicks. Frankfurt/M.: Fischer.

Foucault, Michel. 2005a. Die Maschen der Macht. In: Daniel Defert/François Ewald (Hrsg.), Analytik der Macht. Frankfurt/M.: Suhrkamp, 220-239.

Foucault, Michel. 2005b. Omnes et singulatim. Zu einer Kritik der politischen Vernunft. In: Daniel Defert/François Ewald (Hrsg.), Analytik der Macht. Frankfurt/M.: Suhrkamp, 188-219.

Foucault, Michel. 2014. In Verteidigung der Gesellschaft. Vorlesung vom 17. März 1976. In: Andreas Folkers/Thomas Lemke (Hrsg.), Biopolitik. Ein Reader. Berlin: Suhrkamp, 88-114.

Lepsius, Oliver. 2020. Vom Niedergang grundrechtlicher Denkkategorien in der Corona-Pandemie. Verfassungsblog v. 06.04.2020. https://is.gd/LBFBYw.

Link, Jürgen. 2003. Normativität versus Normalität. Kulturelle Aspekte des guten Gewissens im Streit um die Gentechnik. In: Martin Stingelin (Hrsg.), Biopolitik und Rassismus. Frankfurt/M.: Suhrkamp, 184-205. 
Preciado, Paul B. 2020. Learning from the virus. ARTFORUM 58/9, May/June 2020. https://is.gd/tbFaOp.

NW3/ISL. 2020. Triage. Behinderung darf kein Kriterium bei Priorisierungs-Entscheidungen sein!. https://is.gd/Bef9OK. 17.08.2020

Sarasin, Philipp. 2003. Zweierlei Rassismus? Die Selektion des Fremden als Problem in Michel Foucaults Verbindung von Biopolitik und Rassismus. In: Martin Stingelin (Hrsg.), Biopolitik und Rassismus. Frankfurt/M.: Suhrkamp.

Sarasin, Philipp. 2020. Mit Foucault die Pandemie verstehen? Geschichte der Gegenwart v. 25.03.2020. https://is.gd/ddsYwG.

Soldt, Rüdiger/Wiegel, Michaela. 2020. Wer über 75 Jahre alt ist, wird nicht mehr intubiert. FAZ.NET. https://is.gd/XENZat.

Sotiris, Panagiotis. 2020. Ist eine demokratische Biopolitik möglich? LUXEMBURG ONLINE. https://is.gd/rHnUEU.

Streeck, Hendrik 2020: Infection fatality rate of SARS-CoV-2 infection in a German community with a super-spreading event. medRxiv v. 02.06.2020. https://doi.org/ 10.1101/2020.05.04.20090076. 